\title{
Staat und Kirche auf Titelblättern von Bibelausgaben
}

Exemplarische Beobachtungen in der Bibelsammlung der WLB

Die Relevanz und Akzeptanz von Bibelausgaben wird bis in das frühe 20. Jahrhundert hinein häufig unterstrichen durch Bezugnahmen auf politische Verantwortungsträger bzw. territorial definierte Zielgrößen. Dies geschieht vor allem durch Widmungsvorreden sowie gedruckte oder auf Einbände geprägte Widmungsbilder bzw. Wappen. Seltener sind Andeutungen zum Verhältnis von Staat und Kirche auf Titelblättern von Bibelausgaben. Im Verlauf des 20. Jahrhunderts treten gesellschaftliche Gruppen oder Strömungen als Begründungsbasis für die Relevanz bzw. als Ziel-Objekt der Gestaltung von Bibelausgaben in den Vordergrund.

Das von der anglikanischen Kirche traditionell bevorzugte Modell einer Staatskirche betont die Einheit geistlicher und weltlicher Macht in der Person des Monarchen. Die Bibel begründet und begrenzt nicht die staatliche Macht, wirkt nicht als Korrektiv. Sie wird zumal in ihrer volkssprachlichen Fassung vielmehr verstanden als Gnadengabe des Königs an sein Volk. Das wird am deutlichsten im illustrativen Teil des Titelblatts der sogenannten Great Bible von 1539/1541 (Bb engl. 1541 01). Sie wird anders als die Coverdale-Bibel von 1535, deren Neuausgabe sie darstellt, offiziell im Auftrag des englischen Königs Heinrich VIII. (1491-1547) herausgegeben. Die Übersetzung Williams Tyndales (1526ff.) wird zwar ausgiebig benutzt, aber als Werk eines Kritikers des englischen Königs nirgendwo erwähnt. Die Botschaft des Titelblatts von 1541 ist klar: Der König veranlasst und autorisiert die Bibelübersetzung, gibt die Volksbibel an geistliche und weltliche Amtsträger und - über diese vermittelt - an sein ganzes Volk weiter. Bibelverbreitung und geistliches Wohl des Volkes liegen zwar im Verantwortungsbereich des Königs. Aber beides ist nicht von der politischen Loyalität der Untertanen bzw. von der unmittelbaren Identifikation von Volk, Land, König und Kirche zu trennen. "Es lebe der König" (Vivat rex) bzw. "Gott schütze den König" (God save the king) sind wichtiger als das Lob Gottes.

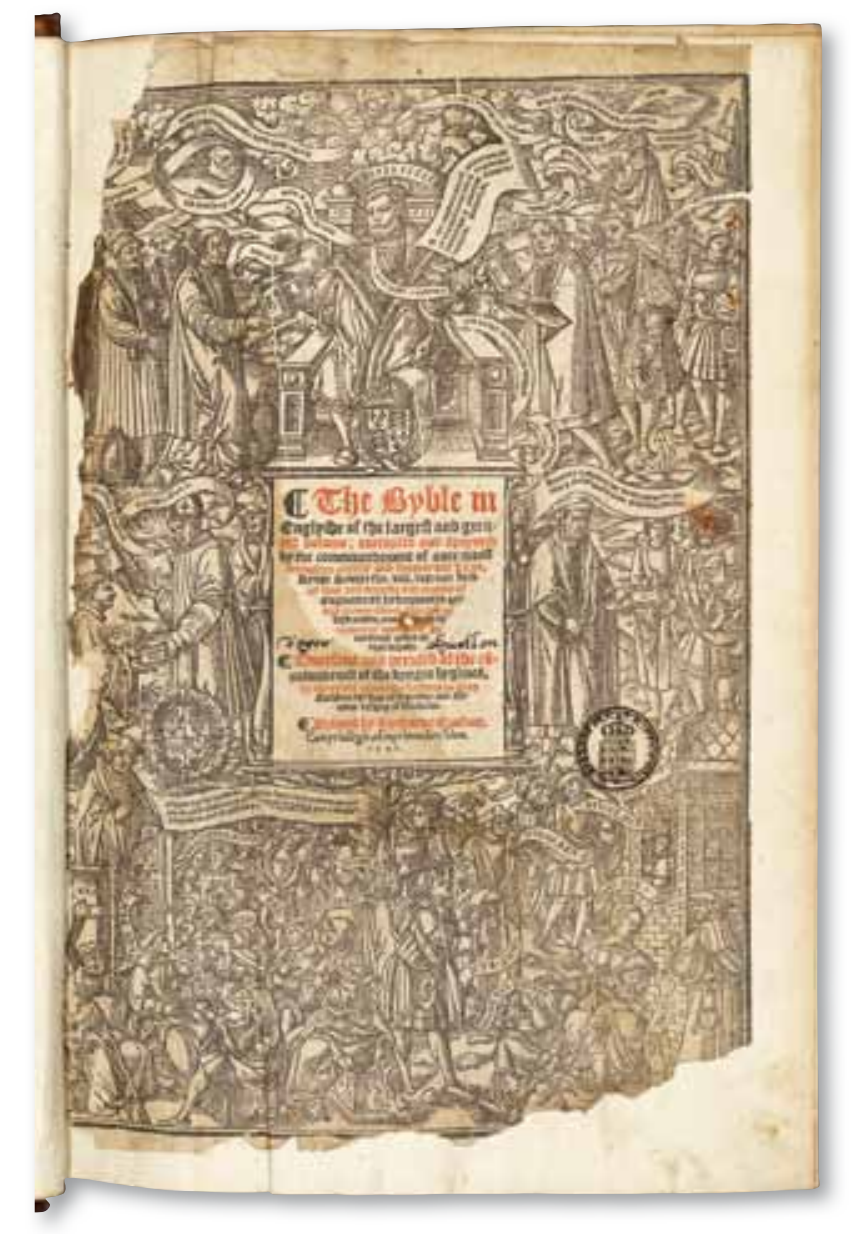

Abb. 1: Bb engl. 154101

Im Investiturstreit durchfochten und im Kirchenstaat exemplarisch konkretisiert, vertritt die katholische Theologie traditionell einen genau umgekehrten, nämlich von der geistlichen Seite ausgehenden Einheitsgedanken von Staat und Kirche. Um die Authentizität der Lehre und den Bewegungsspielraum der Kirche zu erhalten, wird diese tendenziell dem Staat übergeordnet. Weil man der Bibelverbreitung wegen auf das Wohlwollen weltlicher Fürsten angewiesen ist, kann man dieses Staatsverständnis allerdings nur indirekt auf Titelblättern von Bibelausgaben andeuten. So begegnen in der Kölner Dietenberger-Bibel von 1584 (Bb deutsch 1584 01) auf gleicher Ebene als mit ihren Wappen präsente Autorisierungsinstanzen zwei weltliche und drei geistliche Fürsten. In der Titelformulierung kommt 
eine gestufte Motivation der Herausgabe dieser Bibel zum Ausdruck: "Gott zu lob, der Roem. Keis. Maiest. zu allergnedigstem wolgefallen, gemeyner Teutscher Nation zu guetem". Dem weltlichen Herrscher kommt allenfalls eine rechtliche, aber keine inhaltliche Verfügungsgewalt über die Bibelausgabe zu. Er kann sie nicht veranlassen oder korrigieren, sondern sollte ihre Verbreitung unterstützen. Den vier Bilderpaaren mit biblischen Szenen ist das Thema Gehorsam in unterschiedlichen Kontexten gemeinsam. Bemerkenswert ist die Gegenüberstellung der Verehrung des neugeborenen Christus durch die Weisen / Könige aus dem Morgenland und des Kindermords in Bethlehem auf Geheiß des Königs Herodes. Es geht um die Frage, ob man als König der Ehre Gottes dient oder sich gegen die Heilsgeschichte stemmt. Das Schöpfungsbild zwischen den Wappen der weltlichen Fürsten deutet die Begrenzung politischer Macht auf den unmittelbar kreatürlichen Bereich an.

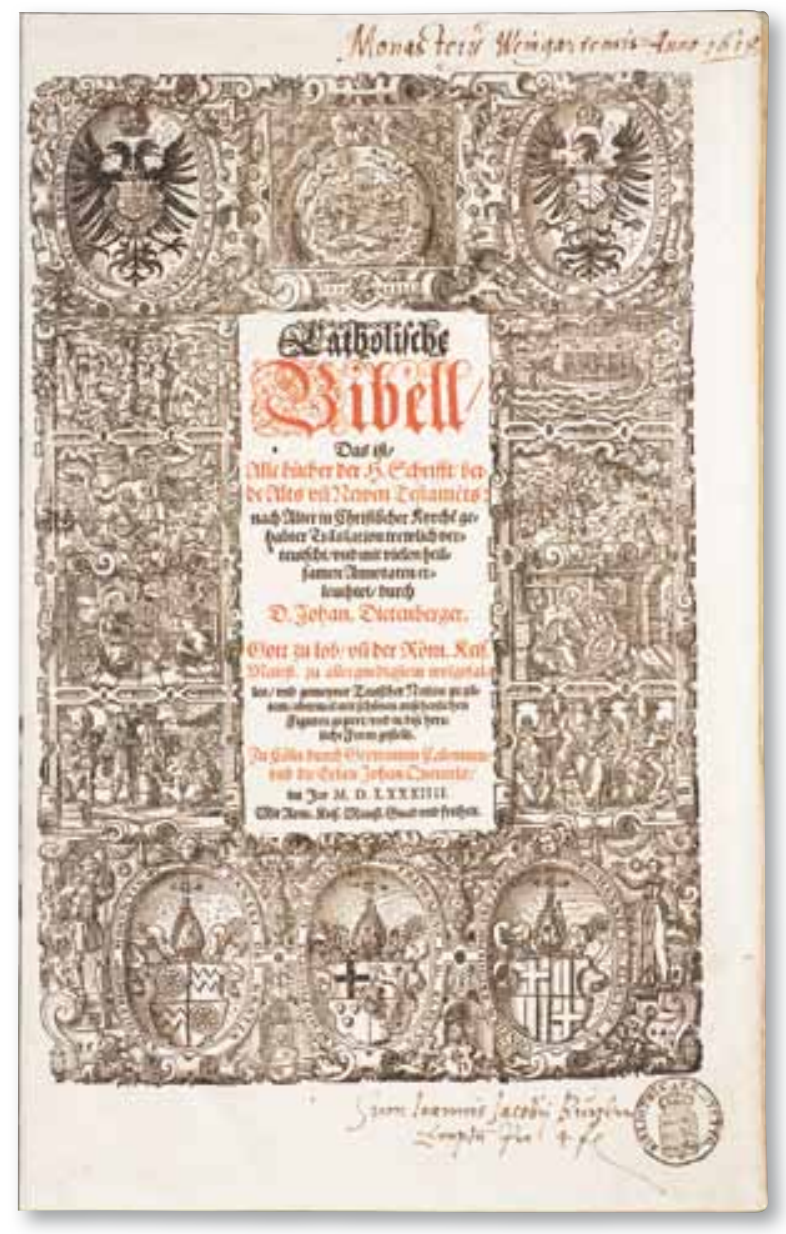

Abb. 2: Bb deutsch 158401

Der Protestantismus wird in Deutschland meist über seinen antiklerikalen Affekt und die zur katholischen Theologie gegenläufige Tendenz zur
Trennung von Staat und Kirche wahrgenommen. Dabei handelt es sich jedoch um eine Verkürzung auf sein liberal-neuprotestantisches Segment. Das in eher konservativen Strömungen fortgeführte Staatsverständnis der lutherischen Zwei-ReicheLehre propagiert nämlich keine Trennung, sondern eine Unterscheidung und gleichzeitige Zuordnung von Staat und Kirche. Ausgangspunkt der Verhältnisbestimmung ist dabei das Reden und Handeln Gottes. Ausgewählte biblische Szenen in einem Bilderzyklus auf dem Titelblatt einer Frankfurter Lutherbibel von 1585 (Bb deutsch 1585 01) verdeutlichen die Sündhaftigkeit und Erlösungsbedürftigkeit des Menschen und den anhaltenden Heilswillen Gottes. Fürsten sind nicht weniger auf Gott - und sein Wort! - angewiesen als ihre Untertanen. Interessant ist die dem Titelblatt folgende Bildseite, die herausragende Vertreter der ernestinischen Dynastie wie der Wittenberger Theologie als Zeugen der Taufe Jesu durch Johannes zeigt. Zwei-Reichebzw. Zwei-Regimenten-Lehre bedeutet: Staatliche wie kirchliche Amtsträger beziehen sich auf das Handeln Gottes zurück, sollen Zeugen, Bekenner, Förderer der biblischen Botschaft sein, allerdings in einer jeweils spezifisch anderen Art und Weise. Staatliche Aufgabe ist zwar nicht die Verkündigung des Evangeliums, wohl aber der Schutz der Freiheit kirchlichen Handelns und der Bibelverbreitung.

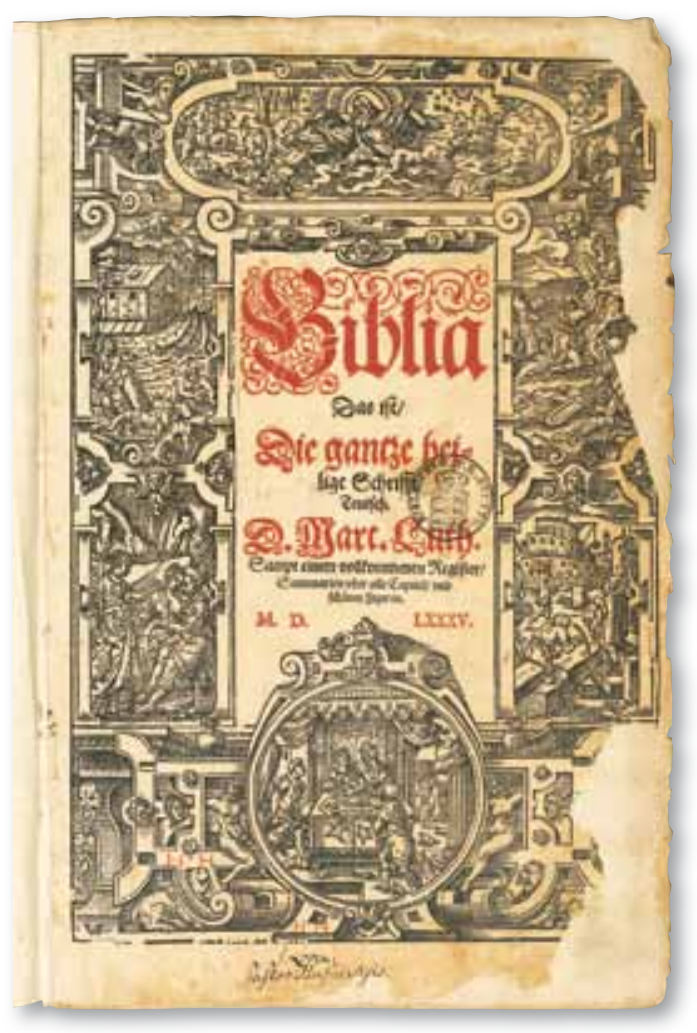

Abb. 3: Bb deutsch 158501 


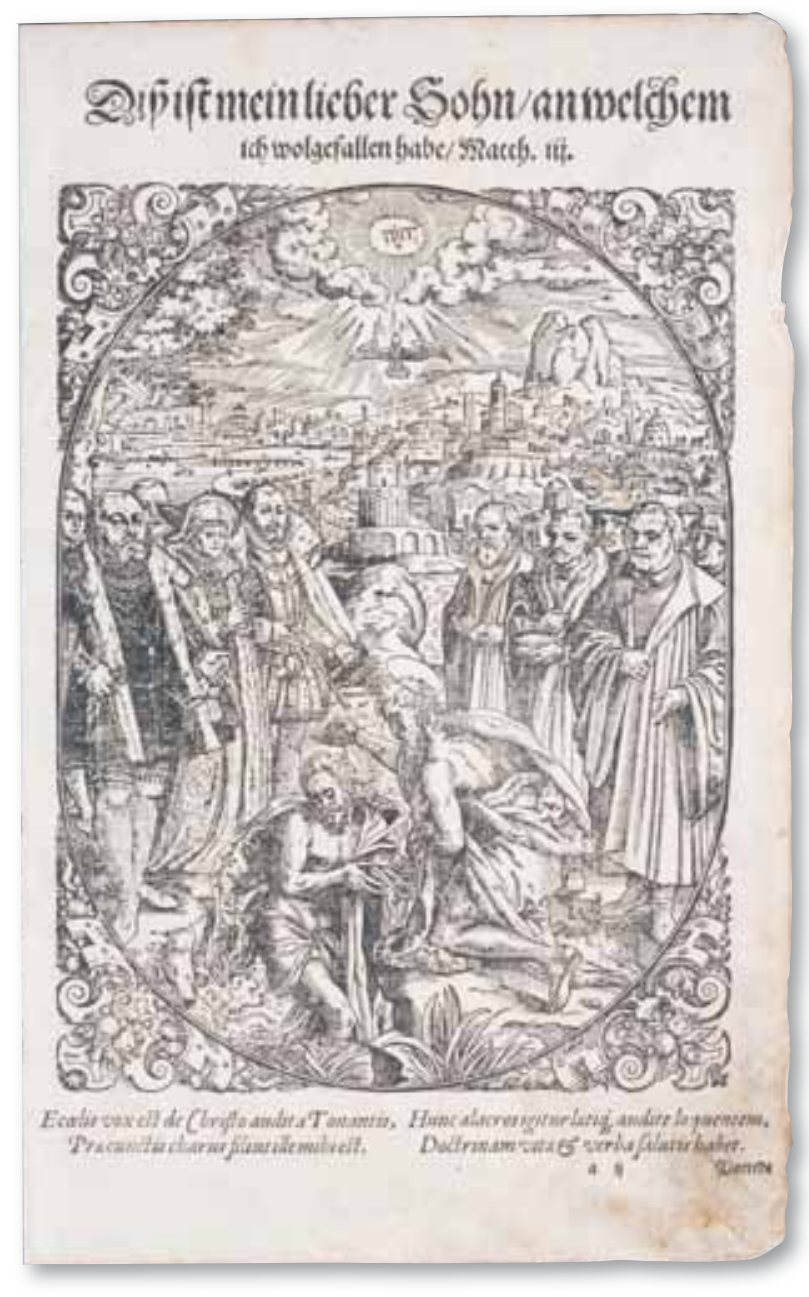

Abb. 4: Bb deutsch 158501

Die Weimarer Kurfürstenbibeln des Nürnberger Verlags Endter sind bekannt für ihre großformatigen Herrscherbildnisse samt Lebensläufen (z.B. $\mathrm{Bb}$ deutsch 1649 01). Die gegenwärtigen Herrscher werden an die sie verpflichtende Traditionslinie der Ernestiner erinnert, die dem Luthertum und der Bibelverbreitung zugewandt waren. Der Obrigkeit kommt ein in Auftrag und Vollmacht begründeter Vorrang gegenüber den Bürgern zu. Das Titelblatt betont hingegen die universale Relevanz der Christusverkündigung. An ihrer Kleidung erkennbare Fürsten wie Bürger stehen um den sie lehrenden Christus und sind mit den Gerechten des Alten und Neuen Bundes versammelt. Im Angewiesensein auf die Offenbarung besteht die Gleichheit der Person vor Gott bei Wahrung innerweltlicher Standesunterschiede. Das Gemeinwohlprinzip christlicher Sozialethik überträgt diesen theologischen Zusammenhang in politisches Handeln.

Der mit dem Haus Endter konkurrierende Verlag Stern in Lüneburg bringt ebenfalls zahlreiche

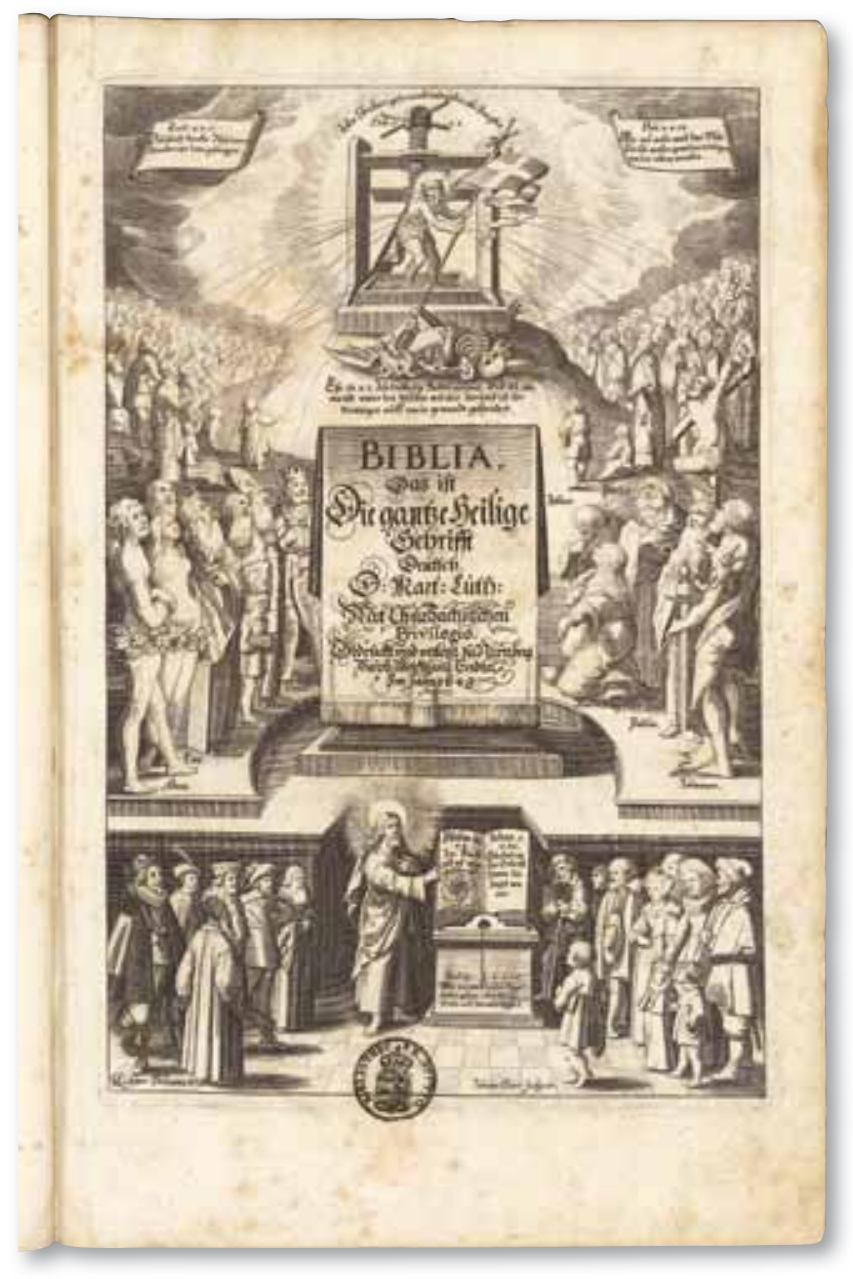

Abb. 5: Bb deutsch 164901

großformatige Lutherbibeln heraus. Dem lutherischen Ansatz entsprechend führt der Kupfertitel der Ausgabe von 1641 (Bb deutsch 1641 01) dem staatlichen Amtsträger als Person die positiven Auswirkungen der Frömmigkeit auf politisches Handeln vor Augen. Tugenden eines Herrschers werden durch figürliche Allegorisierungen vor Augen geführt. Maiestas (Majestät) und Potestas (Macht) stehen zentriert um Pietas (Frömmigkeit; hier mit Wappen). Darüber erscheint das Motto "Regna firmat pietas" (wohl: Frömmigkeit festigt / stärkt Herrschaften). Iustitia (Gerechtigkeit) und Gratia (Gnade) werden illustriert anhand der biblischen Figuren Noah und Johannes der Täufer, der auf Christus verweist. Im oberen Mittelfeld begegnet uns eine Darstellung der Schöpfung vor dem Sündenfall, wohl als Hinweis auf den Zusammenhang von Frömmigkeit und Frieden. Philosophische Tugenden erhalten eine neue, in der Heilsgeschichte fundierte Begründung und theologische Ausrichtung. 
Lutherische Theologie betont das individuell-konkrete „Für dich / mich" der Verheißung bzw. des Heilsgeschehens. Übertragen auf politische Impli-

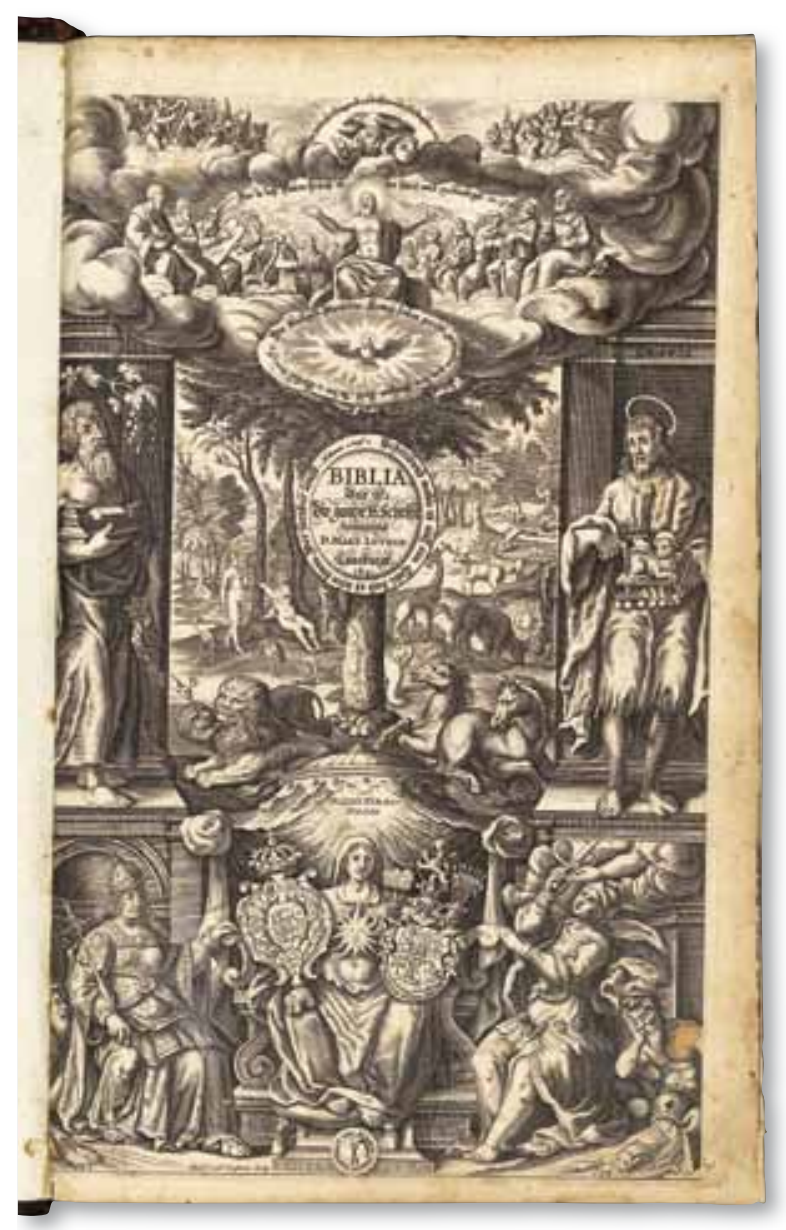

Abb. 6: Bb deutsch 164101

kationen von Bibelausgaben, kann die regionaldynastische Verortung bzw. der konkrete Haftpunkt in der Person eines bestimmten Herrschers als Identifikationsgröße für das betreffende Territorium betont werden. Das geschieht z.B. in der ersten in Württemberg gedruckten Bibelausgabe. Das zweite, kolorierte Exemplar der Bibelsammlung stammt aus dem Besitz Herzog Ludwigs (1554-1593) (Bb deutsch 1590 04). Im ersten Band folgt dem Titelblatt eine Widmungsseite mit dem Porträtbild Herzog Ludwigs, das von allegorischen Tugenddarstellungen umgeben ist. Der Kupfertitel des zweiten Bandes bildet den Herzog zusammen mit einer wohl Martin Luther darstellenden Figur, kniend vor dem gekreuzigten Christus, ab. Die kleineren Bilder auf den Titelblättern nehmen biblische Szenen auf, in denen es um die Erfahrung Gottes als Schöpfer und Erhalter (Band 1) bzw. als Erlöser geht (Band 2). Hinzu kommt das württembergische Wappen im ersten Band. Aussageintention ist: Württemberg und sein aktueller Herrscher sind Gegenstand und Instrument des Wirkens Gottes und zwar hier und jetzt. Eine schwedische, lutherische Bibel von 1655 (Bb schwed. 1655 01) drückt die regionale Verortung bzw. Vergegenwärtigung der universal
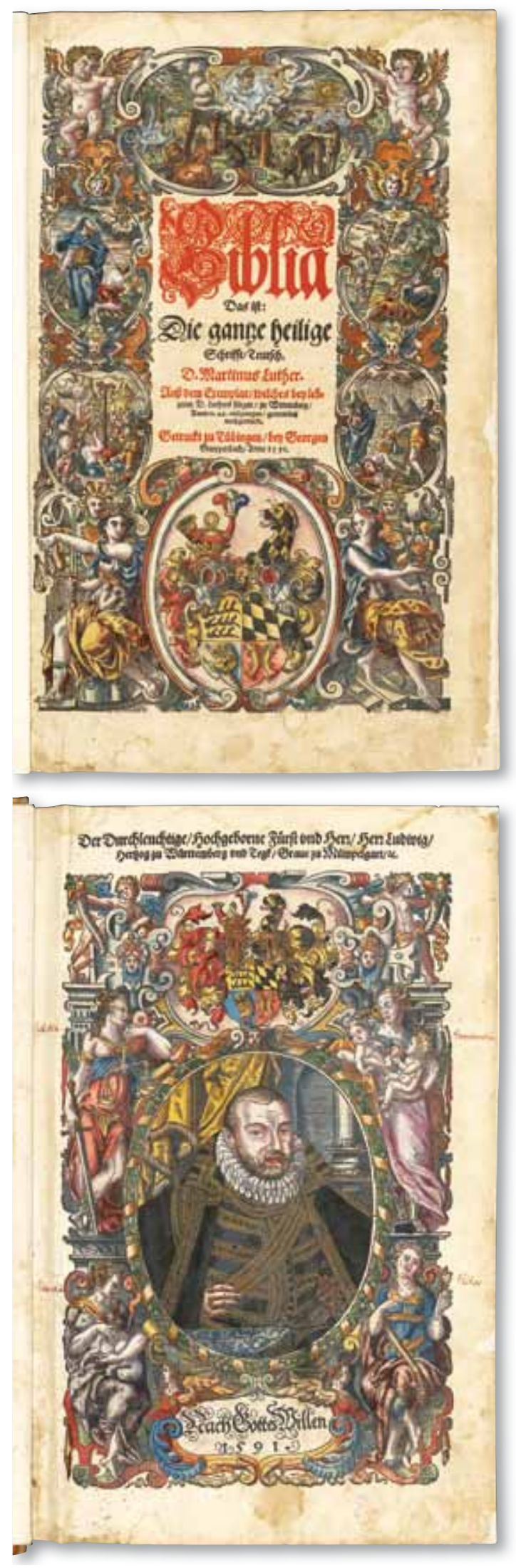

Abb. 7-8: Bb deutsch 159004 
relevanten Botschaft in anderer Weise aus. Neben dem Wappen sieht man eine Stadtansicht von Stockholm mit besonderer Hervorhebung der Stadtkirchen als Orten der Verkündigung.

Typisch für den Protestantismus ist: Erstens wird Universalität durch die inhaltliche Botschaft und die in ihrem Wesen liegende Wirksamkeit der Bibel, weniger durch Amt und Institution begründet. Zweitens lassen sich territoriale Haftpunkte leichter mit nationaler Identifikation und Selbst- vergewisserung verbinden. Das wird nirgendwo deutlicher als bei der mitten im Ersten Weltkrieg erschienenen Kaiser-Wilhelm-Bibel (Ba graph. 1917 01). Der Monarch prägt den Individualnamen der Bibel, ist aber auch durch ein Bildnis sowie Zitate präsent. Es geht quasi um den Vollzug eines obrigkeitlichen Seelsorge- und Trostamtes einerseits, andererseits um die Steigerung des Durchhaltevermögens im Krieg.

Christian Herrmann

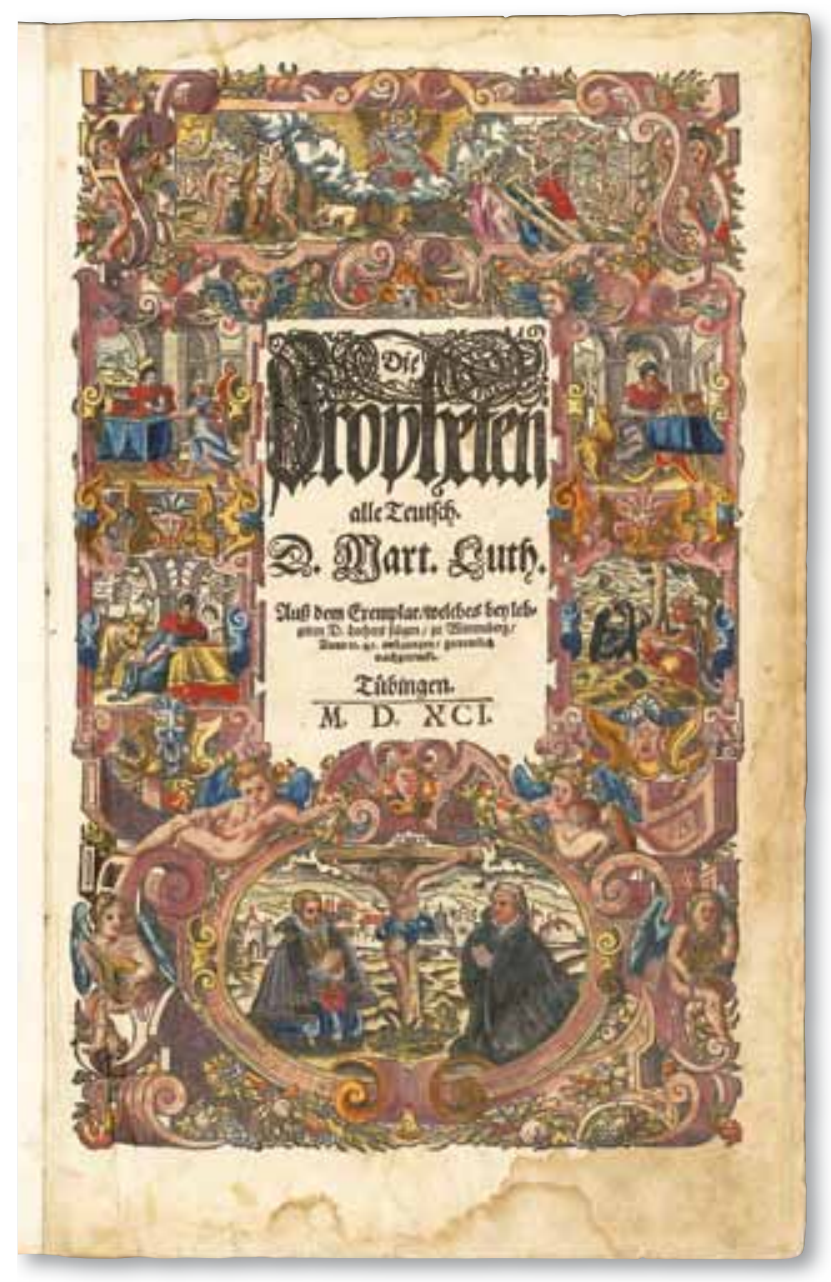

Abb. 9: Bb deutsch 159004

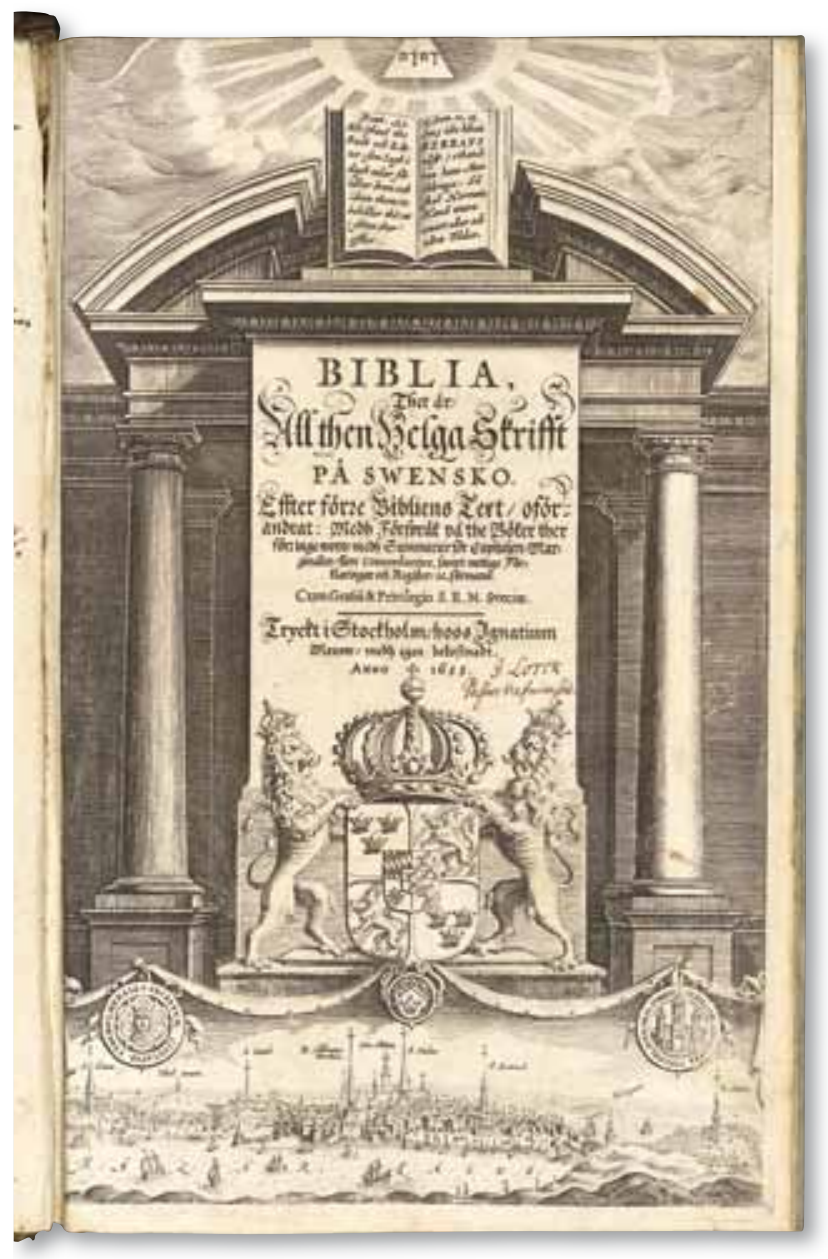

Abb. 10: Bb schwed. 165501 